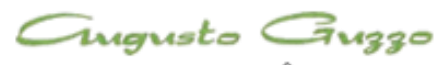

REVISTA ACADÉMICA

\title{
Criando e usando web service
}

\author{
João Alexandre Magri' \\ Recebido em: 06/01/2013. Aprovado em: 21/03/2013. Disponibilizado em: 26/06/2013 \\ 1. João Alexandre Magri é Mestre em Engenharia. Professor e Coordenador do curso de Sistemas de Informação das Faculdades \\ Interadas "Campos Salles".
}

\section{Resumo}

As aplicações projetadas para serem utilizadas via internet são chamadas de aplicações Web. Elas são executadas em um ambiente distribuído onde cada elemento da aplicação pode estar localizado em diferentes computadores conectados via internet. As aplicações Web também são chamadas de sites. Um servidor Web é um software que permite a execução de uma aplicação Web. O computador que armazena o software servidor Web também é chamado de servidor Web, isto é, o software que ele armazena é o que o torna "especial" definindo o seu nome. O computador usado para acessar a aplicação Web é chamado de cliente. A interface desta aplicação é implementada através de um programa chamado do browser (navegador), portanto o usuário utiliza o browser para solicitar serviços e receber as respostas do software Web server. Existem situações em que o cliente nem sempre é um browser. Pode ser, por exemplo, outro programa escrito usando-se a linguagem Visual C\#. Neste caso se tem dois computadores trocando dados sem o uso de um navegador, isto é, o cliente é um programa escrito em Visual C\#. No processamento distribuído os computadores precisam trocar dados: o problema é que eles são representados em cada máquina usando-se formatos não padronizados o que inviabiliza a troca de dados entre máquinas. Existem várias tecnologias para o desenvolvimento de aplicações distribuídas. Uma delas é a Web Server que permite a troca de dados entre dois computadores conectados via internet ou intranet. Basicamente um Web Server é uma classe cujos métodos podem ser chamados a partir de métodos localizados em outras máquinas usando-se um protocolo de comunicação padronizado.

Palavras-chave: Web Service, Visual C\#, Web Server.

\begin{abstract}
Applications that are designed to be used via the Internet are called Web applications. They are executed in a distributed environment where each element of the application can be located on a different computer. Web applications are also called sites. AWeb server is a software that allows a user to run a Web application The
\end{abstract}


computer that stores the Web server software is also called a Web server. The computer used to access the Web application is called the client. The interface is implemented through a program called the browser (browser). The user request services and receive responses from the Web server software through the browser. The client is not always a browser. It may be, for example, another program written in Visual C \# language. In this case there are two computers exchanging data without the use of a browser. When the data processing is distributed among different computers they need to exchange data. If the data in different machines use non-standard formats it will make the exchange of data between machines imposible. There are several technologies for developing distributed applications. One is the Web Server that enables the exchange of data between two connected computers either via the Internet or intranet. Basically a Web Server is a class whose methods can be called from methods located on other machines using a standardized communication protocol.

Keywords: Web Service, Visual C\#, Web Server. 


\section{O que é um Web service}

Serviço é um tipo específico de bem que se caracteriza por ser intangivel, isto é, sem existência física. Um produto também é um bem, porém tem existência física, portanto não é um serviço.

O fornecimento de energia elétrica, o preenchimento da declaração de imposto de renda, a consulta a um médico são exemplos de serviços em geral prestados por uma empresa ou pessoa. Alguns destes serviços podem ser automatizados. Por exemplo, o cálculo do frete para e envio de um produto comprado via internet. $\mathrm{O}$ cliente entra no site da empresa, monta o pedido e através do seu CEP é possível calcular o valor do frete usando-se um programa que está armazenado em um computador que pode ser acessado via internet, isto é, este programa para o cálculo de fretes presta este serviço para o site de compras, ou seja, é um programa que presta serviços para outros programas. Este tipo de programa é chamado de Web service (serviço Web).

A comunicação com os Web service foi padronizada o que possibilitou criar Web services independentes da plataforma usada no desenvolvimento dos programas que solicitam a execução do serviço. Esta padronização foi definida usando-se o protocolo HTTP na transmissão de dados e de um formato de representação de dados baseado na linguagem de marcação XML. HTTP e XML são tecnologias padronizadas pelo W3C (Word Wide Web Consortium) que podem ser usadas em qualquer ambiente de programação.

Nestas condições um sistema para a reserva de passagens aéreas cujos programas foram escritos usando-se a linguagem Visual C\# da Microsoft pode acessar a princípio um Web service escrito na linguagem Java.

\section{Linguagens declarativas ou de script}

As linguagens de programação podem ser classificadas em dois grandes grupos:

- Linguagens declarativas ou de script

- Linguagens procedurais ou imperativas

As linguagens declarativas ou de script exigem que o programador forneça uma descrição exata da tarefa a ser realizada sem a necessidade de explicar como executar a tarefa. As linguagens SQL, HTML, XML, SMIL, LINQ são exemplos de linguagens declarativas.

As linguagens procedurais ou imperativas exigem que o programador escreva um roteiro que especifique detalhadamente como executar uma tarefa. Em geral um roteiro é descrito usando-se um conjunto de comandos possíveis de serem executados por um computador. Estes roteiros são chamados de programas: eles são escritos usando-se uma linguagem chamada de linguagem de programação. As linguagens Visual C\#, Visual Basic, Java, COBOL, $\mathrm{C}++$ são exemplos de linguagem de programação imperativas.

Nestas condições um programador escreve um programa somente quando ele usa linguagens procedurais. Quando ele usa linguagens declarativas ele está descrevendo detalhadamente os resultados esperados para um programa chamado interpretador. $\mathrm{O}$ interpretador é um programa projetado para gerar todo o código necessário para a produção dos resultados descritos pelo programador quando usa uma linguagem declarativa. Nestas condições, as linguagens declarativas são usadas para executar serviços com determinadas características enquanto que as linguagens procedurais são 
de uso geral, isto é, podem ser usadas para a produção de qualquer resultado.

A grande vantagem das linguagens declarativas é que elas são otimizadas para a execução de serviços específicos: nestes casos elas são mais claras e mais poderosas do que as linguagens procedurais. Elas têm um nível de abstração mais elevado do que as linguagens procedurais porque $\mathrm{O}$ programador não precisa especificar os detalhes de execução: feita a descrição precisa dos resultados a serem obtidos o interpretador gera o programa necessário para produzi-los automaticamente e ativa a sua execução.

Nestas condições, para que um computador possa executar a seguinte tarefa: "apresentar um texto em uma página da web" foi criada a linguagem declarativa HTML (Hypertext Markup Language) (Linguagem de Marcação de Hipertexto). Sua primeira versão foi publicada em 1992. Atualmente ela é uma linguagem declarativa padronizada pelo W3C.

Todas as linguagens de marcação são baseadas na linguagem SGML (Standart Generalized Markup Language) que foi criada por TIM Berner Lee, o idealizador da WWW, em 1986. Esta linguagem permite executar a seguinte tarefa: composição e apresentação de documentos na web. A SGML tem recursos suficientes para permitir a especificação e o manuseio de documentos complexos, porém ela é demasiadamente complexa quando usada na composição e apresentação de textos correntes. Assim ela foi simplificada e a nova versão foi chamada de HTML. Esta linguagem foi criada especificamente para:

- Estruturação de documentos correntes,

- Apresentação de documentos em um brawser (navegador), ou seja, a composição e apresentação de documentos na web.

Usar a linguagem declarativa HTML é equivalente a usar um editor de textos que não tenha um menu de comandos nem botões de formatação do texto do documento. Em vez de selecionar o texto e clicar em um ícone para apresentá-lo em negrito, por exemplo, deve-se escrever uma marca especial no início da frase para habilitar a apresentação em negrito e no seu final para desabilitar o negrito. Estas marcas recebem o nome de tag ou "etiqueta de marcação". $\mathrm{Na}$ figura 1 tem-se a evolução cronológica da linguagem HTML.

\section{SGML (1986) $\rightarrow$ HTML 1.0 (1992) $\rightarrow$ HTML xx $\rightarrow$ HTML 4.01 (1999) $\rightarrow$}

XML $1.0(2000) \rightarrow$ XML 1.1 (2006)

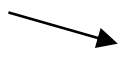

XHTML 1.0 (2002) $\rightarrow$ HTML 5.0 (2009) 
O modelo de hipertexto suportado pela linguagem HTML é muito simples possibilitando uma linguagem fácil de usar. Entende-se por hipertexto a organização e estruturação de informações de uma maneira que se aproxima da forma como as pessoas pensam, estabelecendo relações (links ou ligações) entre uma informação e outra. Nestas condições, um conjunto de documentos expressos na linguagem HTML, isto é, páginas da web constitui um hipertexto porque são apresentados usandose recursos de multimídia, tem interatividade com o leitor e as páginas podem ter vínculos (links ou ligações) entre si dispensando a sua leitura seqüencial como no caso da leitura de um livro.

\subsection{Introdução à linguagem XML}

Atualmente a maioria dos documentos que são transmitidos via web são codificados usando-se a linguagem de marcação ou de publicação HTML. Um dos seus pontos fortes é a sua simplicidade o que permite seu uso em várias aplicações web.

Com a evolução das técnicas de projeto das aplicações web surgiram novos requisitos difíceis de serem atendidos usando-se a linguagem HTML. Visando suportar estas novas necessidades muitos fabricantes de browsers (navegadores) começaram a introduzir novos recursos em seus produtos o que provocou a perda do padrão HTML fazendo com que as páginas de um site pudessem ser executadas por determinado browser e em outros não.

Visando eliminar este processo o W3C definiu outra linguagem padronizada chamada XML (eXtended Markup Language) que atendia às novas necessidades, isto é, garante a sua independência dos vários navegadores disponíveis no mercado. Como no caso da linguagem HTML a linguagem de marcação XML também é baseada na linguagem SGML e foi projetada para complementar a linguagem HTML e não para substituí-la. Ela foi projetada para estruturar, armazenar e transportar dados com foco no significado dos dados enquanto que a linguagem HTML foi projetada para apresentar (imprimir) os dados com foco na aparência ou modo com que estes dados são apresentados.

Um documento XML apenas empacota um conjunto de dados. Nada é executado com a linguagem XML, portanto é necessário escrever um programa para o envio, recepção e impressão dos dados. $\mathrm{Na}$ figura 2 tem-se um exemplo do conteúdo de um arquivo cujo conteúdo está representado na linguagem XML.

$<$ ?xml version="1.0" encoding="ISO-8859-1" ? $>$
$\quad$ CATALOG $>$
$<$ CD $>$
$<$ TITLE $>$ Status quo live $</$ TITLE $>$
$<$ ARTIST $>$ Status quo $</$ ARTIST $>$
$<$ COUNTRY $>$ Inglaterra $<$ COUNTRY $>$
$<$ COMPANY $>$ Vertigo $</$ COMPANY $>$
$<$ PRICE $>$ 40.90 $</$ PRICE $>$
$<$ YEAR $>\mathbf{1 9 7 6}</$ YEAR $>$
$</$ CD $>$
$<$ CD $>$
$<$ TITLE $>$ Greatest Hits $</$ TITLE $>$
$<$ ARTIST $>$ Canned heat $</$ ARTIST $>$




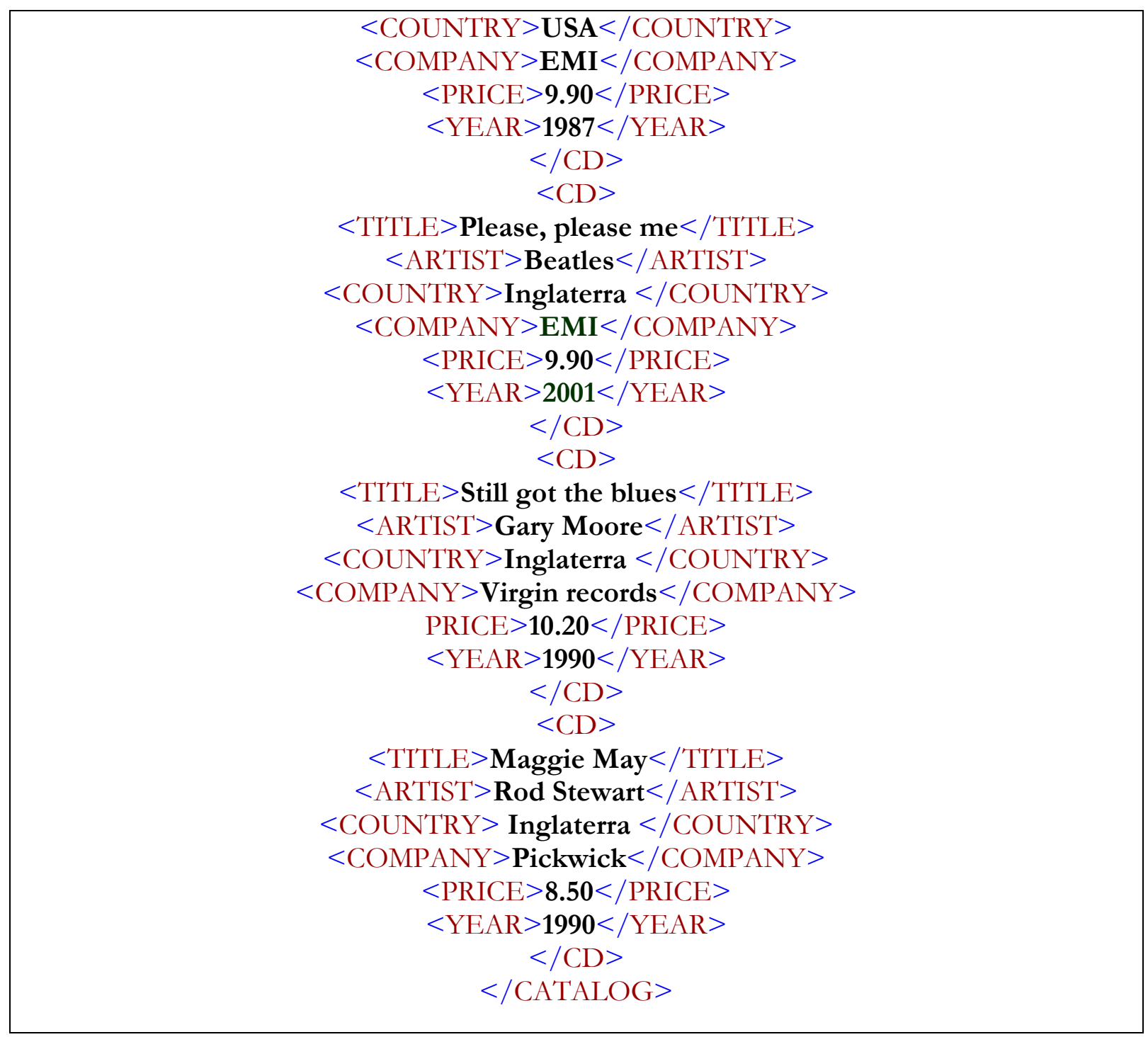

Figura 2 conteúdo de um arquivo expresso na linguagem XML

A linguagem $\mathrm{XML}$ tem as seguintes vantagens:

a) simplifica a troca de dados entre unidades de processamento de dados.

Em geral cada fabricante de software usa um formato próprio para a representação dos dados: um arquivo XML é padronizado e armazena os dados usando o tipo texto o que garante a sua independência do software e do hardware de qualquer equipamento, portanto é possível a troca de dados entre dois sistemas usando-se arquivos gerados no padrão XML. A linguagem foi criada para permitir a interação de aplicações escritas em linguagens diferentes e para facilitar a comunicação entre aplicações.

b) O padrão XML aumenta a disponibilidade dos dados porque eles podem ser acessados a partir de fontes de dados XML e não somente a partir de páginas web. 
c) A linguagem XML pode ser usada para a criação de outras linguagens a serem usadas na web.

Esta é uma das vantagens mais importantes desconhecida por muitas pessoas. A partir da linguagem XML foram criadas as seguintes linguagens:

- XHTML

- WSDL: usada para descrever como usar um Web service

- WAP e WML: são linguagens de marcação usadas em dispositivos móveis

- SMIL: usada para a autoria de documentos hipermídia

- RSS: trata-se de um formato padrão que permite a divulgação de notícias ou novidades de um site ou blog. Para isso o link e o resumo da notícia são armazenados em um arquivo com extensão XML. Este arquivo é chamado de feed ou feed RSS. O conteúdo dos feeds, isto é, as notícias podem ser lidos por um programa ou serviço leitor de RSS chamado agregador. Nestas condições é possível ter conhecimento das novidades de um site sem ter que acessá-lo a procura das suas novidades.

d) A linguagem XML permite separar os dados do HTML

Esta vantagem permite revisar rapidamente o HTML quando os dados mudam.

\subsection{Introdução à linguagem XH'TML}

A linguagem XHTML resultou da combinação das linguagens HTML e XML. A especificação da linguagem XML é muito rígida o que não ocorre com a linguagem HTML.Assim quando os navegadores (browsers) encontram a tag $<\mathrm{p}>$ por exemplo, sem estar fechada com o respectivo $</ \mathrm{p}>$ no HTML este erro é consertado automaticamente pelo navegador, com os seus recursos o que significa: aquilo que funciona em um dispositivo pode virar uma bagunça em outro. Com o XHTML tem-se a garantia de que o site (conjunto de páginas XHTML) será exibido conforme foi projetado em qualquer navegador (browser) ou dispositivo.

O XML permite criar elementos de marcação e atributos, isto é, pode-se criar uma linguagem de marcação assim, as tags do XHTML foram criadas considerando-se as tags da linguagem HTML versão 4.01 e suas regras. Nestas condições, escrever um código usando a linguagem XHTML significa escrever um código expresso em XML onde as tags e atributos já estão definidos e são aquelas existentes na linguagem HTML.

A linguagem XHTML apresenta as seguintes vantagens em relação à linguagem HTML:

a) O código é limpo e estruturado o que facilita o serviço de manutenção da aplicação

b) O tempo de carregamento de uma página XHTML é menor porque o navegador interpreta uma página limpa sem ter que corrigir erros

c) Uma página XHTML é compatível com páginas HTML antigas e com as aplicações dos usuários em geral

Basicamente o XHTML é uma forma mais restritiva de montar uma aplicação escrita em HTML de tal forma que o código seja um XML válido.

A principal característica da linguagem XHTML é a acessibilidade, isto é, uma 
página XHTML pode ser acessada pelo Explorer, Firefox, Opera, Netscape, Safári, Chrome, televisão, celular, etc., isto é, qualquer recurso.

\subsection{Introdução à linguagem H'TML5}

Conforme pode ser visto na Figura 1 o HTML 4.01 publicado em 1999 pelo W3C é a última versão da linguagem. No início de 2009 o W3C anunciou a primeira especificação da versão 5 . O lançamento final que estava previsto para 2012 foi adiado para o segundo trimestre de 2014 porque $\mathrm{O}$ W3C pretende atender às necessidades de vários perfis de usuários. No processo de atualização da linguagem foram introduzidos os seguintes recursos:

- Novas APIs uma delas para o desenho de gráficos tridimensionais,

- Manipulação de conteúdos multimídia,

- Melhor depuração de erros.

Uma das novidades mais importantes é a eliminação de plug-ins para aplicações multimídias em navegadores tais como o Flash da Adobe, Silverlight da Microsoft, JavaFX da Oracle, etc. O principal destaque da linguagem HTML5 é o avanço na programação de vídeos.

A maioria dos navegadores do mercado já implementaram a nova versão do HTML. Ela representa um grande avanço em relação à versão anterior e poderá ser a linguagem mais utilizada no serviço de implementação das aplicações web a serem desenvolvidas nos próximos anos.

\section{Protocolos usados em um} ambiente de processamento que utilize um Web service
No mercado estão disponíveis várias linguagens de programação tais como Visual C\#, Java, Perl, Python, F\#, etc. As aplicações escritas usando-se estas linguagens não podem se comunicar porque cada linguagem tem o seu modo padrão para o tratamento dos dados a serem processados. Um Web service é uma das soluções para se resolver este problema de comunicação através do uso de uma combinação de protocolos não proprietários (abertos) e padrões.

Para que se possa usar um Web service para resolver este problema, é necessário atender os seguintes requisitos:

1. Definir um padrão para a troca de dados entre aplicações,

2. Definir um padrão para o envio e recepção de mensagens entre aplicações,

3. Definir um padrão para descrever as características de um Web service,

4. Criar um dicionário que permita executar uma pesquisa visando encontrar determinado Web service na internet.

\subsection{Transferência de dados entre aplicações}

Para a transferência de dados entre aplicações é usada a linguagem XML que foi projetada para esta finalidade.

\subsection{Envio de mensagens para um Web service e recebimento da resposta}

O envio e recepção de mensagens entre aplicações, isto é, mensagem para ativar a execução de um Web service e receber o resultado do processamento é feito usandose o protocolo SOAP (Single Object Access Protocol) ele permite a independência da plataforma onde o Web Service estiver 
rodando e da linguagem de programação usada na implantação do Web service.

Outro protocolo que tem uma função equivalente ao SOAP é o HTTP: ele é usado para acessar páginas e navegar na internet sem que o usuário precise saber qual é o servidor web (IIS da Microsoft, Apache, etc.) nem a linguagem usada na codificação da página acessada (HTML, XHTML, ASP.NET, etc.).

O SOAP tem as seguintes características:

. é baseado em XML para a troca de informações em um ambiente distribuído,

. Foi definido pelo W3C,

- Usa HTTP como protocolo de transporte,

- É um padrão de utilização com Web service.

\subsection{Descrição das características de um Web service}

Um cliente pode usar um Web service desde que ele conheça: service,

. Qual a URL de acesso ao Web

. Nome do Web service,

- Descrição de cada método, isto é, seu nome e a lista de parâmetros a serem passados, isto é, sua assinatura,

. Como o cliente e o serviço devem processar a requisição.

Estas informações são fornecidas em um arquivo cujo conteúdo é expresso na linguagem WSDL (Web Service Description Language). Ela é uma linguagem baseada em XML, padronizada pelo W3C e usada para descrever as características de em Web service.

\subsection{Dicionário para a localização de um} Web service na internet

Após montar um Web service deve-se publicá-lo na internet, isto é, tornar o Web service disponível para os usuários. A publicação de um Web service pode ser feita através de um serviço de diretório chamado UDDI (Universal Description, Discovery and Integration) que é mantido pelas empresas que atuam no mercado de software tais como Microsoft, Oracle, IBM, Intel, SAP, etc, além de grandes empresas do mercado em geral. Atualmente a comunidade UDDI está composta por cerca de 370 empresas. A versão 3.0 foi declarada como padrão em 2005.

\section{Características de um Web service}

Um Web service tem as seguintes características:

. São projetados para permitir a troca de dados entre diferentes plataformas e a reutilização de software,

- A comunicação com os Web services é feita usando-se protocolos abertos,

. São independentes da linguagem de programação usada na sua implantação,

. Podem ser localizados usando-se o diretório UDDI,

- Podem ser usados por qualquer aplicação. A aplicação pode ter sido escrita em uma linguagem diferente daquela usada para implementar o Web service,

- a linguagem XML é usada na implementação das características citadas anteriormente, 
- Sua implantação é simples e de baixo custo porque usa a infraestrutura de redes já existente,

. Reduz significativamente os custos de integração de aplicações e de comunicação B2B (Business to Business),

. São implementados utilizando-se o ciclo de vida incremental e interativo reduzindo os custos $\mathrm{e}$ as dificuldades de implementação de uma tecnologia radicalmente diferente.

\subsection{Diferenças entre um Web service e um Web site}

O uso de Web services permite criar um modelo abrangente de desenvolvimento de sistemas que pode ser implementado em qualquer sistema operacional que suporte a comunicação via web, portanto é comum comparar um Web service com um Web site. As principais diferenças entre eles são:

- A aplicação web é projetada para interagir com os seus usuários enquanto que os Web services são projetados para interagir com outros programas (aplicações),

- Nas aplicações web tem os programas navegadores (browsers) como clientes enquanto que os Web services tem qualquer tipo de cliente ou dispositivo,

As aplicações web tem uma interface enquanto que os Web services não tem.

Nestas condições, estes dois tipos de aplicação rodam na web, porém foram projetadas para atender a requisitos diferentes.

\section{Criação de um Web service}

Um Web service é uma classe que permite a chamada dos seus métodos a partir de métodos localizados em outras máquinas via protocolos e formatos de dados padronizados tais como HTTP e XML. No ambiente de desenvolvimento .NET a chamada de um método através da internet é feita usando-se o protocolo SOAP. Opcionalmente pode-se usar a arquitetura REST (Representational State Transfer) que está baseada no tradicional mecanismo requisição / resposta.

O conceito de Web service foi definido pela Microsoft a partir do ano 2000 quando a empresa adotou os Web services como sendo o componente principal da sua recém lançada plataforma .NET. Inicialmente todo o trabalho para o desenvolvimento das ferramentas necessárias para a implementação de um Web service foi dirigida para os detalhes funcionais relativos ao envio e recepção de mensagens codificadas no padrão SOAP.

Estas ferramentas tornaram a implementação dos Web services bastante simples porque boa parte do código necessário é gerada automaticamente na plataforma .NET.

Basicamente pode-se criar um Web service adicionando a um projeto ASP.NET um arquivo com a extensão .asmx via Visual Studio ou iniciar um novo projeto específico para um Web service.

\section{Exemplo:}

Criar e consumir um Web service chamado WSCalculoTaxas que contém dois métodos:

$$
\begin{aligned}
& \text {. imposto (float renda) } \\
& \text {. receber (float renda, float desco) }
\end{aligned}
$$

O valor do imposto deverá ser calculado a partir da seguinte tabela (fictícia): 


\begin{tabular}{|l|l|}
\hline Renda & Percentual de desconto \\
\hline$<=3.400,00$ & 10 \\
\hline$>3.400,00$ & 20 \\
\hline
\end{tabular}

O método imposto $($ calcula o imposto devido em função da renda do funcionário e o método receber() calcula o valor a ser recebido pelo funcionário. Na Figura 3 temse a representação da classe (web service) WSCalculaTaxas segundo a UML (Unified.Modeling Language).

\begin{tabular}{|c|}
\hline WSCalculaTaxas \\
\hline Não tem campos \\
\hline+ imposto(float renda) \\
+ receber(float renda, float desco). \\
\hline
\end{tabular}

Figura 3 Representação da classe WSCalcula'Taxas segundo a UML

Neste caso optou-se por iniciar um novo projeto específico para um Web service.

\section{Passo 1:}

Nestas condições deve-se abrir o Visual Studio 2008 ou superior e selecionar as seguintes opções:

File/New/Project/ ASP.NET Web service application/ nome do Web service: neste caso WSCalculaTaxas / OK

$\mathrm{Na}$ Figura 4 tem-se a janela da opção New Project usada para definir os dados gerais do novo projeto.

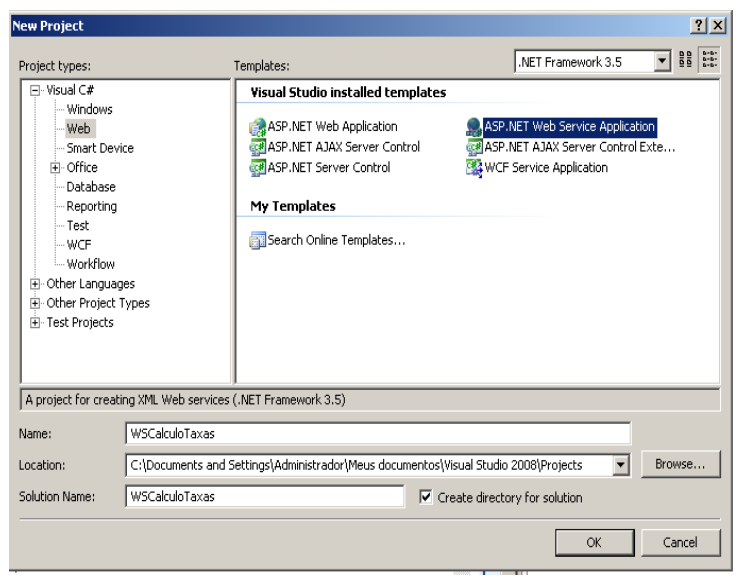

Figura 4 Janela New Project para a digitação dos dados gerais do Web service

$\mathrm{Na}$ figura 5 tem-se a janela Solution Explorer: observar que o Visual Studio criou o arquivo Service1.asmx que contem todo o código gerado automaticamente, conforme já foi explicado.

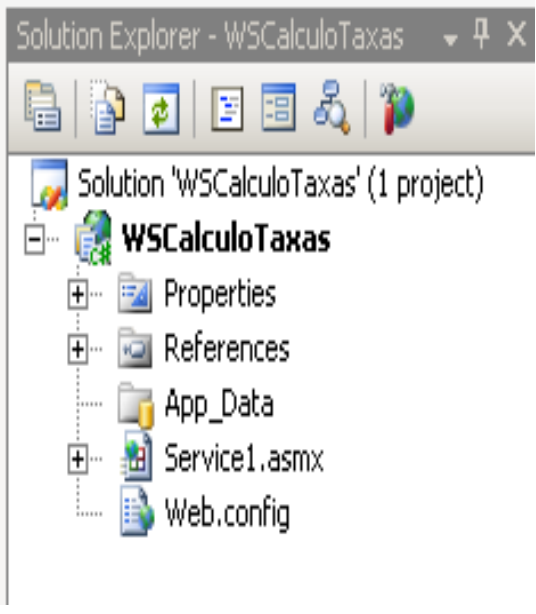

Figura 5 Janela Solution Explorer e o arquivo Service1.asmx gerado automaticamente

\section{Passo 2:}

$\mathrm{Na}$ Solution Explorer dar duplo click na pasta Service1.asmx para abrir o conteúdo desta pasta no editor do Visual Studio. Na Figura 6 tem-se o conteúdo desta pasta. 


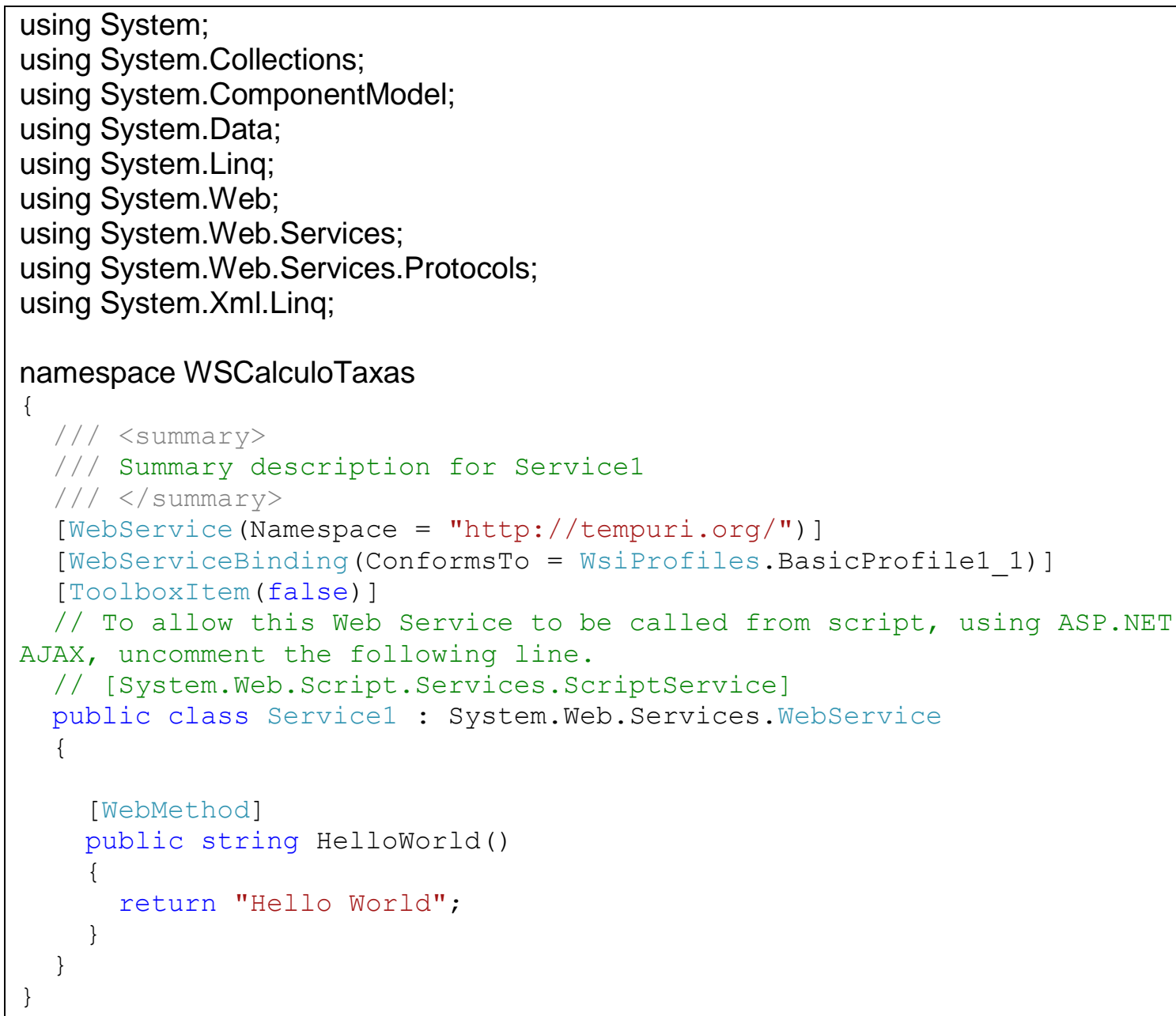

Figura 6 Listagem do arquivo Service1.asmx

Observar que o código é igual ao de uma outra classe qualquer: a única diferença é o atributo [WebMethod]. Pode acontecer que seja necessário bloquear o acesso do exterior a determinados métodos da classe Web service: isto pode ser feito não escrevendo este atributo antes do cabeçalho do método. Por exemplo, na Figura 6 o método HelloWordo pode ser acessado a partir do exterior porque antes do seu cabeçalho foi escrito o atributo [WebMethod].

Todos os métodos da classe Web service devem ser digitados na pasta service1.asmx . Na Figura 7 tem-se esta classe e os métodos da aplicação que está sendo desenvolvida. 


\section{Passo 3 (digite os métodos):}

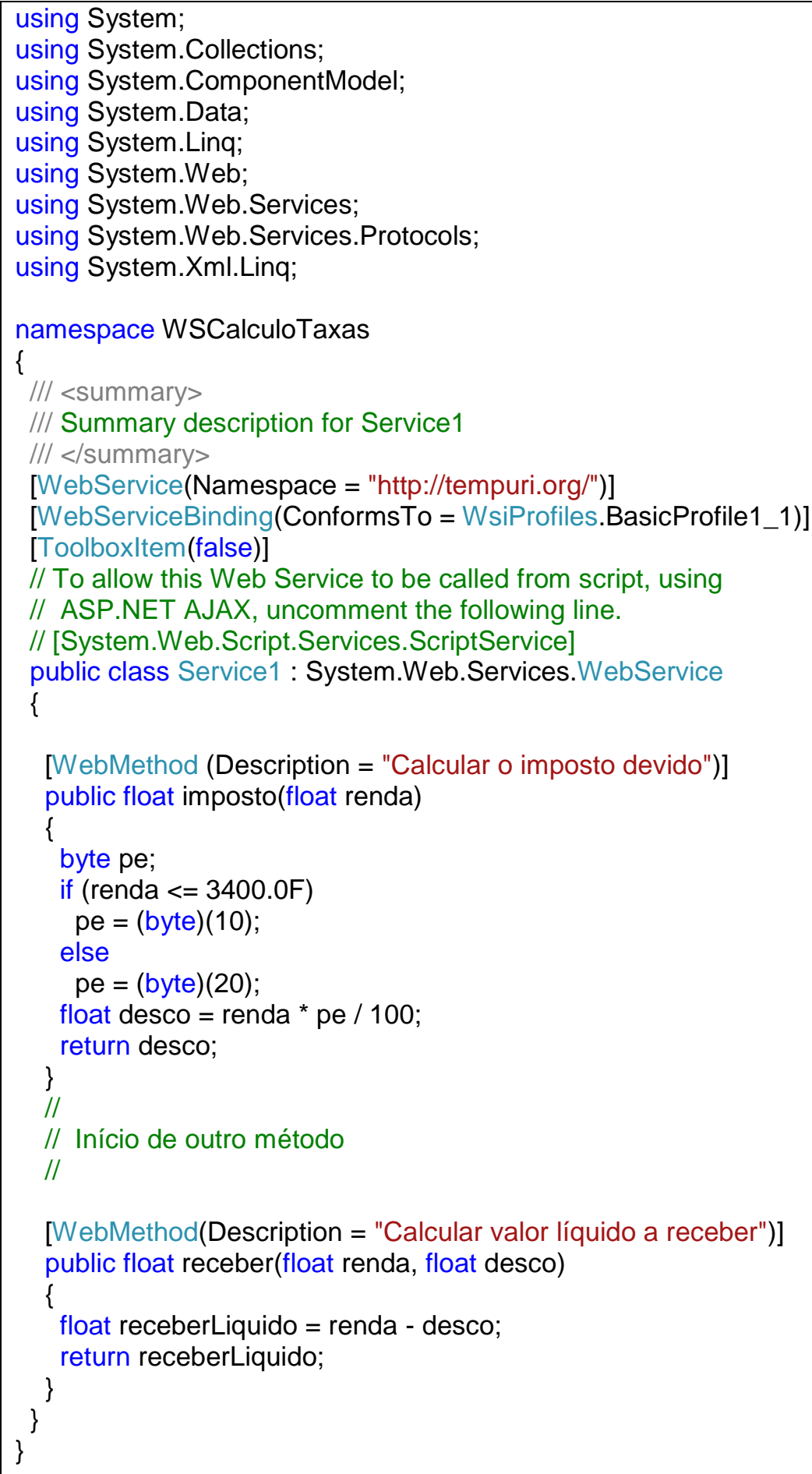

Figura 7 Métodos da classe Web service 
Observar que antes do cabeçalho de cada método foi escrito o atributo [WebMethod], portanto os dois métodos podem ser chamados a partir do exterior. Além disso, este atributo tem o parâmetro Description que permite a inserção de um comentário onde é feita a descrição do serviço que é feito pelo método.

Este projeto não tem interface porque é um Web service, portanto não é preciso criar um formulário e arrastar os objetos de tela para ele.

\section{Passo 4:}

Após digitar todos os métodos da classe WSCalculaTaxas, deve-se verificar se os métodos produzem resultados corretos, isto é, executar o Web service. Para isso pressionar as teclas:

\section{Ctrl F5}

O VS responde com a janela da figura 8

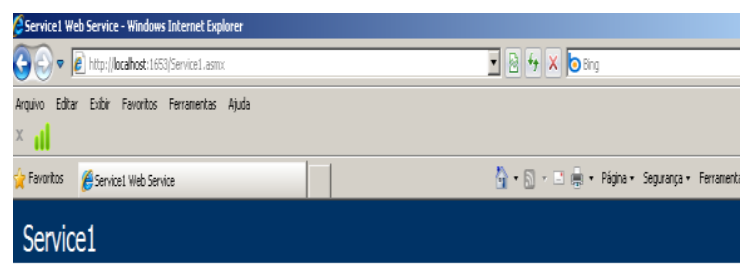

The following operations are suppocted. for a formal defintion, please review the Service Description.

- imposto

Caluaro imposto denito

- receber

Caludar valor liquido a reseber

This web service is using http://tempuri.org/ as its default namespace.

Reconmendation: Change the defaut namespace before the XML Web service is made public.

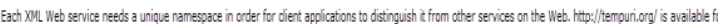

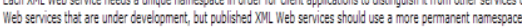

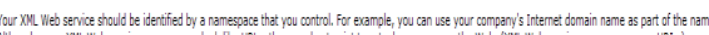

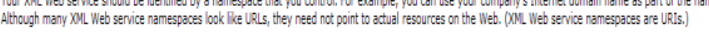

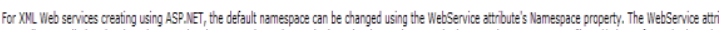

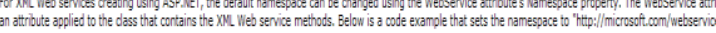
ct

[TebService (Vlanespaces="hitcp://ricrosoft. com/vebservices/")]

public class Ayliebservice

// implenentation

Figura 8 Janela com botões para testar a execução dos métodos do Web servisse

\section{Passo 5:}

Clicar no botão imposto para calcular o valor do imposto usando o Web service. O VS reponde com a caixa de digitação apresentada na figura 9 .

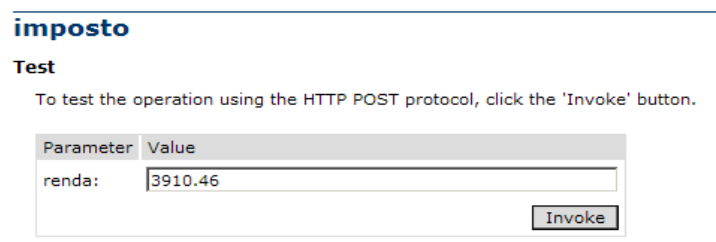

Figura 9 Caixa para digitar o valor da renda do funcionário

Digite o valor da renda e clique no botão Invoque (vide Figura 9). O VS responde com uma página contendo a listagem do arquivo XML que será enviado como resposta para o método solicitante, conforme está mostrado na Figura 10. Observar que o valor do desconto será de $\mathrm{R} \$ 782,092$ sendo do tipo float.

$<$ ?xml version $=" 1.0 "$ encoding $="$ utf $-8 "$ ? >

<float $x$ mlns $="$ http: / / tempuri.org/">782.092 </float $>$

Figura 10 Arquivo contendo o resultado do processamento expresso na linguagem XML

\section{Passo 6:}

Repetir o passo 4 clicando no botão receber e digite os valores da renda e do desconto. Faça o cálculo e verifique se o resultado está correto.

Se os resultados estiverem corretos o Web service está funcionando perfeitamente e seu URL vale http://localhost:1653

A seguir é necessário criar um novo projeto a fim de acessar os recursos ou consumir o Web service WSCalculaTaxas. 


\section{Criação de uma aplicação para consumir o web service criado no item 5}

Este novo projeto poderia ser um web form ou um Windows form. No caso deste exemplo será criada uma aplicação do tipo Windows form e será usada a linguagem Visual C\# para a codificação dos métodos. $\mathrm{O}$ projeto será chamado de consomeWSCalculoTaxas.

Passo 1: Abra o VS e selecione as seguintes opções:

File/New/Project/ Windows Forms application/ nome do projeto: neste caso ConsomeWSCalculaTaxas / OK

Passo 2: a aplicação deve "saber" que deverá acessar um Web service portanto deve ter todos os seus dados, inclusive o URL. Os arquivos que descrevem o Web service já foram gerados automaticamente pelo VS portanto falta "registrar" este Web service nesta aplicação. Este trabalho de "registro" é chamado de referência, portanto deve-se referenciar o Web service WSCalculaTaxas nesta aplicação. Para isso, deve-se selecionar as seguintes opções:

Clicar com o botão direito do mouse no nome do projeto: no caso consomeWSCalculaTaxas / Add web reference / Digitar o URL que foi definido pelo VS (ver Figura 8) / clicar no botão GO $\mathrm{O}$ VS responde com a janela da figura 11

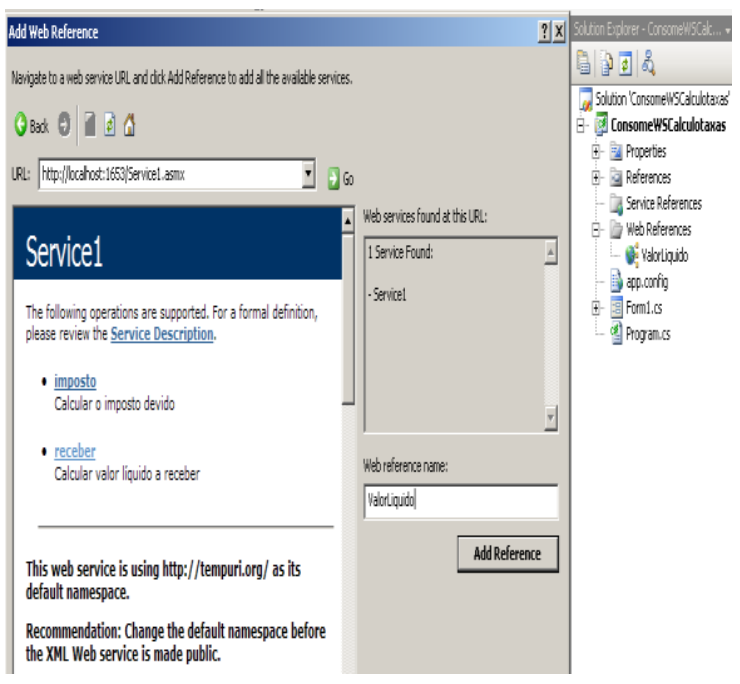

Figura 11 Janela para referenciar um web service e fornecer o nome da referência

Passo 3: Selecionar as seguintes opções:

Clicar no botão Advanced / clicar no botão Add a web reference / digitar o nome da referência. Neste caso será ADOTADO o nome: Valor Liquido / clicar no botão adicionar

Passo 4: montar o formulário de acordo com a figura 12 .

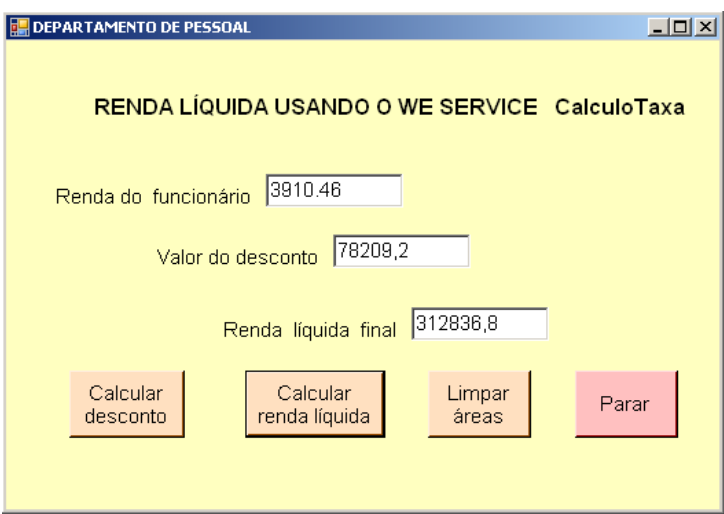

Figura 12 Lay out da interface da aplicação que acessa o Web service

$\mathrm{Na}$ figura 13 tem-se a solution Explorer desta aplicação. Observar que existe uma referencia a um web service (Service 
References) que corresponde à referência do WSCalculoTaxas chamada Valor Liquido.

\section{Solution Explorer - ConsomeWSCalculotaxas

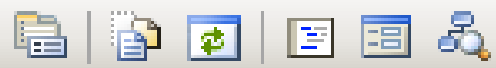 \\ Solution 'ConsomeWSCalculotaxas' (1 F

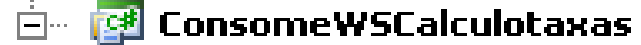

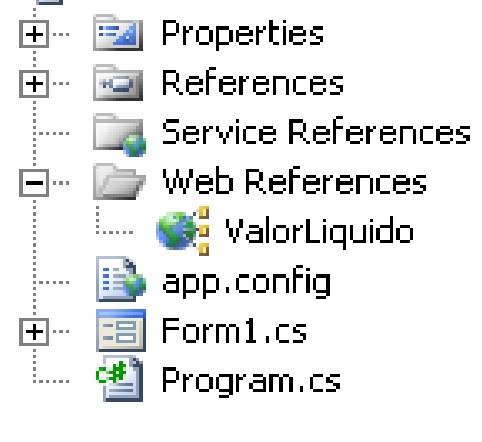

Figura 13 Solution Explorer da aplicação que consome o Web service

Passo 5: Digitar o código dos métodos de acordo com a listagem 1.

\begin{tabular}{c} 
using System; \\
using System.Collections.Generic; \\
using System.ComponentModel; \\
using System.Data; \\
using System.Drawing; \\
using System.Linq; \\
using System.Text; \\
using System. Windows.Forms; \\
namespace ConsomeWSCalculotaxas \\
\{ \\
public partial class frmRendaLiquida : Form \\
public frmRendaLiquida() \\
\{ \\
InitializeComponent(); \\
\} \\
private void cmdCaDesconto_Click(object sender, EventArgs e) \\
\{ \\
ValorLiquido.Service1 objWSCalculoTaxa = new \\
ConsomeWSCalculotaxas.ValorLiquido.Service1(); \\
float renda = Convert.ToSingle(txtRenda.Text); \\
float desconto = objWSCalculoTaxa.imposto(renda); \\
txtDesconto.Text = desconto.ToString(); \\
\} \\
\hline
\end{tabular}




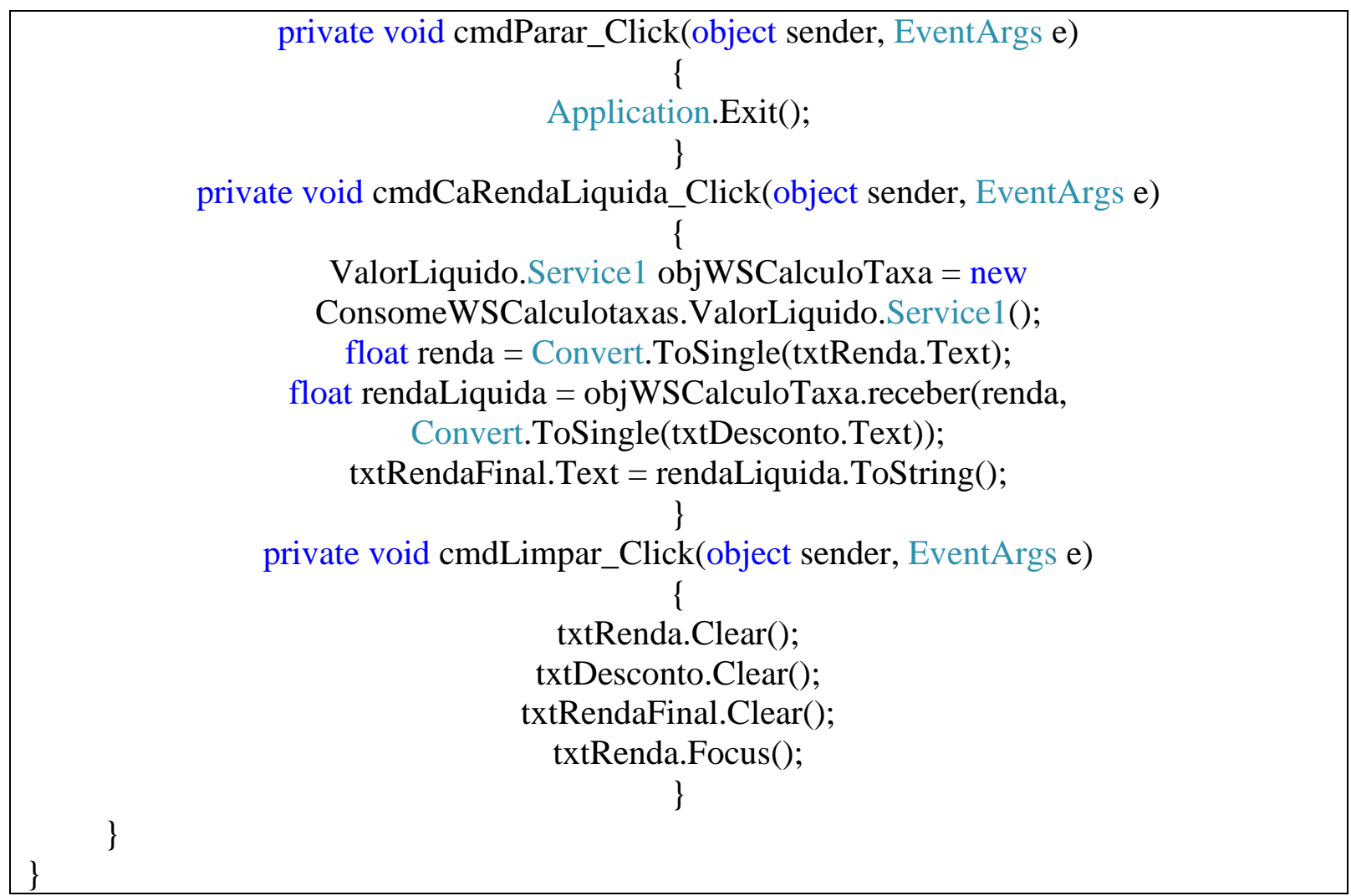

Listagem 1 Código dos métodos de acesso ao web service

Passo 6: compilar esta aplicação selecionando as opções: Bild / Bild solution

\section{Considerações Finais}

No ambiente de desenvolvimento. NET da Microsoft é muito fácil montar um Web service porque o Visual Studio gera automaticamente boa parte do código. O mesmo se aplica no caso da aplicação que consome o Web service.

Os Web services também podem ser usados para a implementação de arquiteturas orientadas a serviços chamadas de SOA (Service-Oriented Architectures). Neste modelo de arquitetura os requisitos principais são definidos como serviços que são acessados por outros serviços aumentando a coesão dos componentes da aplicação.
No próximo artigo será detalhada a montagem de Web services usando WCF (Windows Communication Foundation) que é a nova tecnologia proposta pela Microsoft para o desenvolvimento de aplicações distribuídas.

\section{Referências Bibliográficas}

1. MAGRI, J. A. Programação C\#. 1. Ed. São Paulo: Editora Érica, 2012.

2. MAGRI, J. A. e RODRIGUES, V. B. Princípios da Arquitetura Dirigida a Modelos (MDA). Trabalho de Conclusão de Curso (Graduação em Ciência da Computação). Fundação Instituto Tecnológico de Osasco, São Paulo, 2007.

3. MARGARIE, F. LINQ in action. 1. ed. California: O’ reilly Media Inc, 2008. 
4. NAGEL, C. Professional C\# and .NET 4. 1. ed. Indianapolis: Wiley Publishing, Inc, 2010

5. PIARLOSI, P. Programming Microsoft LINQ in Microsoft .NET framework 4.0. 1. ed. California: O’ reilly Media Inc, 2010.
6. RATTZ, J. Pro LINQ: Integrated Query in C\# 2010. 1 ed. Indianapolis: Wiley Publishing, Inc, 2010

7. SHARP, J. Microsoft Visual C\# 2008. 1. ed. Washington: Microsoft Press, 2008

8. STELLMAN, A. Head First C\#. 1. ed. California: O’ reilly Media Inc, 2008. 